\title{
Research on Information Integration Oriented Supply Chain of Telecom Value-added Service
}

\author{
Guoli Wang and Shoulian Tang \\ Economics and Management School, Beijing University of Posts and \\ Telecommunications, Beijing 100876, P.R. China \\ wg1996@tom.com tangshoulian@263.net
}

\begin{abstract}
This paper discusses information integration oriented supply chain of telecom value-added service. Firstly, it uses a series strategic method. Secondly, it argues how to integrate the supply chain within the web service. Thirdly, the paper describes an example that explains how to manage value-added services for banks. The dynamic system has enabled a more complex type of interaction and collaborations among partners.
\end{abstract}

Keywords: Web service, Parla, Corba, Integration, Dynamic system

\section{INTRODUCTION}

Telecom value-added services (TVAS) have become so important that all enterprises in telecom industry look regard or view their own value-added services as a new source of growth. The ultimate success of a company will depend on how the company constructs, controls and integrates the supply chain formed with its business partners [1].

How to integrate supply chain effectively, how to research services and contents which have no relation to the telecom network and how to make value-added services transferred and manage them more effectively are the theoretical and practical exploring issues. The author attempts to discuss these problems from the perspective of information integration of a value-added services supply chain, and obtain some useful insight.

\subsection{Known Results}

The Web Services technologies are comprised of XML/SOAP/WSDL/UDDI [2]. Web Services establish interoperability of distributed applications for the new platform [3]. Standardization initiatives like those leaded by Parlay or OMA, have been specifying several standard Web Service interfaces to the most common Telco functionalities [2]. 


\subsection{Our Results}

We study information integration oriented supply chain of telecom value-added service. Our solution is that Web services combine with telecom system and traditional CORBA [4] interface is used between application server and Parlay gateway. This kind of Web-based integrated information system is not only loosely coupled, but also can be controlled. Otherwise it is compatible with the former system.

\section{PRELIMINARIES}

\subsection{Problem Description}

Telecom Value-added supply chain has upstream units and downstream units, and especially increasing upstream suppliers. Now, various intelligent application platforms for corresponding services are being used, which brings more and more complex different platforms and interfaces. In the beginning it is possible that telecom value -added supply chain has a number of platforms. But with the technology and business development, there must be a suitable method of integrated supply chain information. Standardization initiatives like those leaded by Parlay or OMA, have been specifying several standard Web Service interfaces to the most common Telco functionalities [2]. But these new solutions could not be perfectly controlled like the former. Traditional Information integration of telecom value-added supply chain is a tight coupling, because the intelligent network integrates it. Different services have different intelligent networks. When intelligent networks have formed a large group in a supply chain, optimization and coordination become very badly, particularly for operators to provide more and more interfaces. Upgrade and management for these platforms are very complex. Furthermore, the heterogeneous information processing needs middleware technology, so researching services has many obstacles. Thus, a tight coupled and controlled system is needed.

\subsection{Web Service}

Web Services establish interoperability of distributed applications for the new platform [3]. Web Service platform is a set of standards which defines how applications on the Web achieve interoperability. Web Service is a service-oriented architecture [2] that defines a set of standard protocols, which is used to define the interface, to invoke methods, to register components, and to achieve various applications based Internet. Currently, there is no exact definition for the Web Services. Generally it is believed that web service is a new type of Web applications, which are self-contained, self-descriptive, and of the modular characteristics that can be published through the Web. Web services can realize the function of a simple 
response to customer requests, and accomplish a complex business processes. Once configured, Web Services can be directly found and used by the other applications and web services. The Web Services technologies are comprised of XML/SOAP/WSDL/UDDI [5], which empowers the Web Services differently from the traditional technologies. Web Services Architecture [3] is a service-oriented analysis and design (Application of OOAD). It is a service-oriented architecture that is used for designing, implementing and deploying the components of a logical development. Figure 1 presents the Web Services Architecture -- Service-Oriented Architecture (SOA) Map.

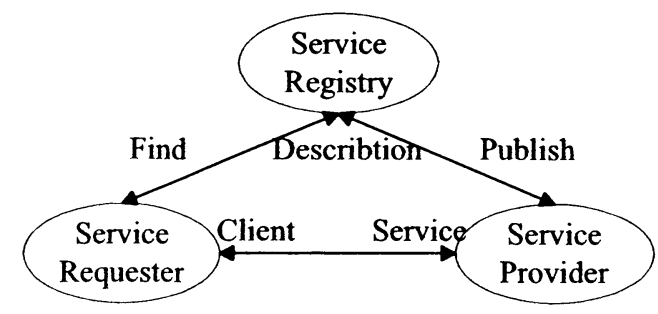

Figure 1. Web Architecture

\section{INTEGRATION OF TELECOM VALUE-ADDED SUPPLY CHAIN}

\subsection{Web-based Information Integration System of Telecom Value-added Supply chain}

Telecom system has several open platforms for value-added systems. The ParlayไOSA system is the most important open system. Web Services combine with the promising open telecom system. Web-based integrated information system is not only a loosely coupled system, but also can be controlled as illustrated in figure 2 .

Application servers are the clients, which implement service logic and search corresponding services from Web service Registration center. Registration Web service center is responsible for the registration and management of Web services. Parlay Web Gateway provides various basic telecom services. 


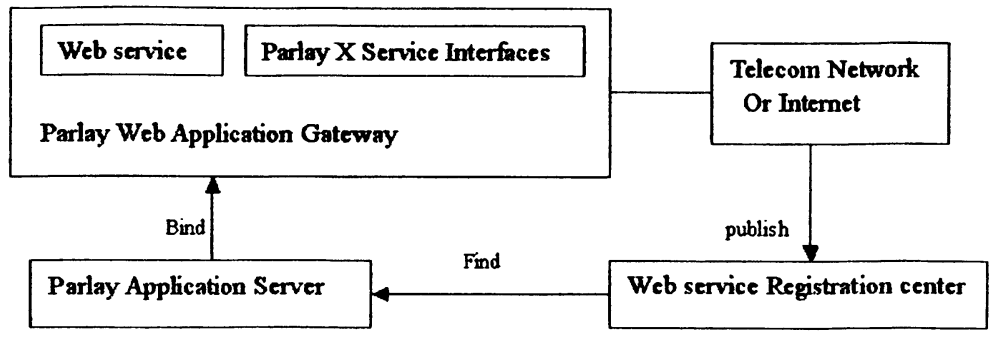

Figure 2. Web-based Open System

\subsection{Prominent Aspects}

Web service will be integrated with Parlay interface and traditional CORBA interface of telecommunications networks. Operator interface is the key for integrating telecom supply chain by Web service. This technology has four prominent aspects.

(1) Easy integration: Services Based on the Parlay Web Services or Parlay X are more easily integrated with Application systems of other enterprise to configure loosely coupled supply chain, to offer services easily and quickly, to enhance the whole value of the supply chain.

(2) Deployment and operation: UDDI of the Web Services can be introduced to the UDDI deployment environment to offer new ways to discover Gateway for service or to publish various network capabilities. Various Parlay Gateways can be registered in the UDDI registration center, and suitable Parlay Gateway can be searched and chosen to access Network.

3) The influence on third-party application and development: Research and Development of TVAS can be comparatively easy because the researchers need not to understand the telecom technology to make use of Web Services application interfaces, particularly the high-level interface such as Parlay X. Web Services that are open and standard, make far-ranging applications easy to access the Gateway.

\section{AN EXAMPLE: BANKCARDS VALUE-ADDED SERVICES}

For the customers using bankcards at services terminal, the services can be controlled as illustrated in figure 3. Parlay Gateway requests these services from Parlay Application Server. After Parlay application server receives the request, it looks up the services in the Web services center. And after services confirmed, it implements the bankcard services logic that is located at the Parlay application server. This logic of the bankcard services uses the bank cards Web Services operation by parlay application services. The Bank Card Web Services operation invokes corresponding operation at Parlay Gateway Interface, sends protocol to the bankcard control center. And the control center takes out user information and requests by 
analyzing the data. Then the control center makes a decision according to the data available in the control center's database. A certain amount of funds may be transferred to the retailer from the customer account and generate corresponding information, which is being sent to the users and stored in a database.

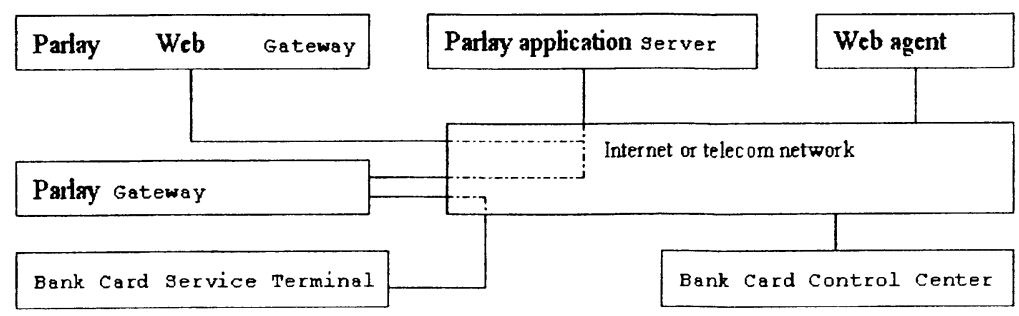

Figure 3. Bankcards Value-added Services Map

\section{CONCLUSIONS}

This paper discusses the information integration of TVAS in the perspective of supply chain and gives some suggestions. A Web-based Information Integration System of TVAS is advanced. The integration of Web services and telecom system is loosely coupled and can be controlled, which do not only meet the requirement of supply chain, but also meet the requirement of telecom management. Finally this paper gives an example of bankcards value added services.

\section{REFERENCES}

1. D.M. Lambert and M.C. Copper, Issues in supply chain management, Industrial Marketing and Management. Volume 29, Number 1, pp.65-83, (2000).

2. Anonymous, Parlay X Web Services Specification, Parlay (June, 2004). http://www.parlay. org/specs/index. asp(Accessed October 6, 2006).

3. G. Alonso, F. Casati, H. Kuno, and V. Machiraju, Web Services: Concepts, Architectures and Applications (Springer: Heidelberg, Berlin, 2004).

4. Anonymous, Common Object Request Broker Architecture: Core Specification, version 3.0.2, Object Management Group (OMG) (June, 2002). http://www.omg.org/cgi-bin/apps (Accessed October 20, 2006).

5. Anonymous, $W 3 C$ Proposed Recommendation, World Wide Web Consortium (W3C) (May 7, 2003). http://www.w3c.org/TR/2003/PR-soap12-part1-20030507 (Accessed January 4, 2007). 